\title{
Real-Time frontal mapping with AUVs in a Coastal Environment
}

\author{
Henrik Schmidt and James G. Bellingham \\ Massachusetts Institute of Technology, Cambridge, MA 02139 \\ Mark Johnson and David Herold \\ Wood Hole Oceanographic Institution, Woods Hole, MA 02543 \\ David M. Farmer and Richard Pawlowicz \\ Institute of Ocean Sciences, Sidney, BC
}

\begin{abstract}
A new oceanographic measurement concept is presented, combining a tomographic network with small autonomous underwater vehicles. Using wireless local area nctwork technology, the acoustic tomography data are recorded and processed in real time for achieving a low-resolution estimate oceanographic parameters. The results are used to focus direct measurements by a network of small AUV's in areas of high spatial and temporal variability. By combining the high coverage but low resolution of the tomography with the high resolution capabilities of the AUVs, oceanographic phenomena with small scale dynamics controlled by large scale environmental forcing can be mapped. The feasibility of the new measurement concept has been demonstrated in connection with a June 96 experiment in Haro Strait, BC, aimed at mapping the properties coastal fronts driven by combined estuarian and tidal forcing.
\end{abstract}

\section{INTRODUCTION}

A fundamental problem facing oceanographic measurement techniques is a trade-off between coverage and resolution. Thus, a measurement system may cover a large area, but only provide course resolution, or provide much higher resolution over a smaller area. This problem occurs in particular in relation to oceanographic phenomenae which cannot be properly described at a single scale, but require consistent and coordinated measurement and analysis at a wide range of spatial and temporal scales. An example is coastal environments, where the estuarian and tidal driven oceanography interacts with medium and small scale topography to create an extremely dynamical oceanography with strong currents and distinct frontal structures. Such frontal structures are the main regions of mixing and upwelling, and are consequently of extreme importance to the coastal ecosystem. They also provide one of the controlling factors for coastal transport processes.

Past studies of such frontal structures have focused either on high resolution volume measurements directly in the frontal region, or larger scale synoptic surface observations, but a measurement system accomplishing both simultaneously has been lacking..

Here we present a new approach to ocean sampling

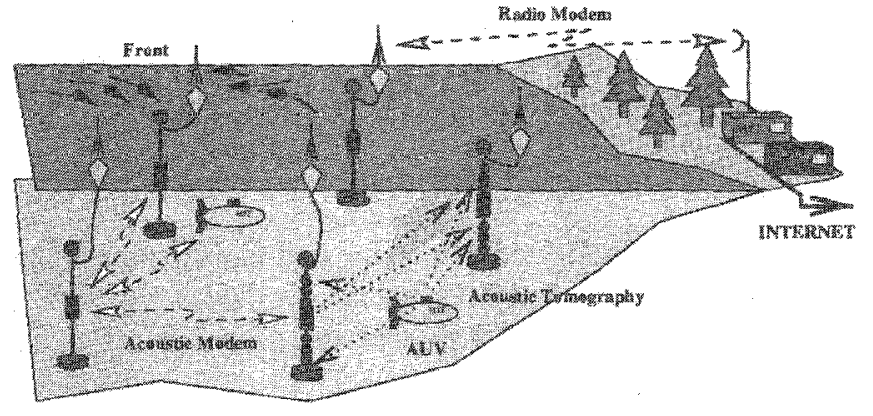

Fig. 1. Hybrid oceanographic sampling network for mapping of frontal dynamics in a coastal environment

which combines the coverage capability of acoustic tomography with the high resolution capability afforded by mobile platforms. In this case the mobile platforms are small autonomous underwater vehicles (AUVs), operating in a moored acoustic communication and navigation network. The tomography component is achieved by adding a vertical hydrophone array to each of the network moorings, Fig. 1.

Once this Hybrid Oceanographic Sampling Network concept is fully developed, the tomographic imaging capability will be used to provide a low-resolution, but close to real time, estimate of the frontal position. Using the acoustic communication network the AUVs are then guided into areas of high spatial variability to perform high-resolution direct measurements of curents, temperature, salinity, and other oceanographic parameters. Thus the fast, low resolution tomography is used to provide coverage while the vehicles provide high resolution.

The feasibility of this new oceanographic measurement concept was demonstrated in a June 96 experiment carried out in Haro Strait, British Columbia. The scientific objective of the experiment was the mapping of a coastal front driven by combined estuarian and tidal forcing. The technological objectives was to determine the performance of the various components of the hybrid sampling network concept in a highly dynamic ecoastal environment, and through coordinated measurements with the various subsystems to determine the feasibility of the hybrid sampling network concept.

Four moorings containing acoustic modems, tomography sources, and 16-element vertical arrays was deployed in the region of the expected frontal activity, S of Stu- 


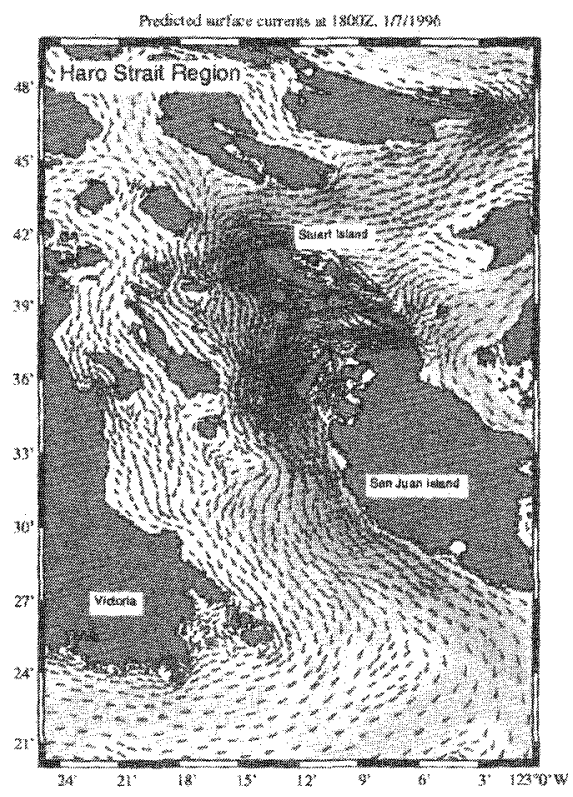

Fig. 2. Topographic map of Haro Strait region, with predicted ebb tide currents during experiment. Tomography array (blue circles) is deployed to investigate the flow convergence or front south of Stuart Island. Current meter moorings (green circles) will help clarify larger-scale circulation, with meteorological data from a surface buoy (green cross).

art Island, Fig. 2. In addition 4 transponders for acoustic navigation of the AUVs were deployed, covering an area of approximately $4 \mathrm{~km}^{2}$. Two Odyssey class AUVs, equipped with CTD, ADCP and Doppler sidescan systems, as well as acoustic tomography and communication sources were operated in the network either individually or in tandem for several hours each day during the experiment. In addition the region was instrumented with various oceanographic instruments, such as moored current meters, drifters and Lagrangian floats.

\section{Haro Strait Oceanography}

Haro Strait and its adjacent channels (Fig. 2) form the most dynamically active portion of the fresh water estuary that extends from the mouth of the Fraser River through Juan De Fuca to the Pacific. It is in this area that tidal mixing is most intense, resulting in modification of the surface waters moving out of the system and also the two-way exchange. Accurate models of the circulation in this area, which includes such important deep water exchange with the Strait of Georgia and surface water flushing around Victoria, requires an adequate representation of the turbulent mixing. Thus far the primary modelling effort has been directed towards meeting the need for tidal current prediction (Foreman et. al, 1995). This model has been extended to a very high resolution within the experimental area, an example of which is illustrated with arrows in Fig. 2 during an ebb tide.

Some of the mixing occurs in highly confined areas such

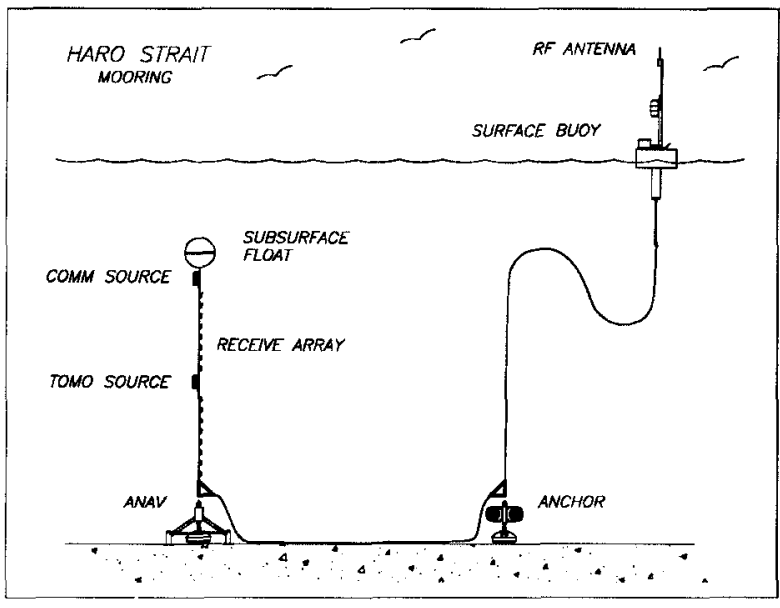

Fig. 3. Acoustic array and mooring configuration in Haro Strait experiment.

as the tidal fronts, for example near Stuart Island (Fig. 2). These fronts may have a well defined surface expression. Some of the mixing evidently occurs in broader areas, possibly due to turbulence caused by the stratified water being driven over shallow regions such as the banks south east of Victoria. Tidal forcing is dominant, although meteorological effects, especially wind conditions over the southern Strait of Georgia, can be important in setting up the stratification at the northern boundary.

We identify the scientific goals as having two components: (i) analysis of the small scale dynamics in intense mixing zones, especially the tidal fronts, and (ii) development of a larger scale model description that incorporates and provides a basis for assessing the relative importance of mixing due to these small structures, in a larger picture of the estuarine and tidally driven circulation.

\section{Oceanographic Sampling Network}

The instrumentation applied to study the frontal dynamics in Haro Strait was a hybridization of traditional oceanographic floats and moorings, drifting instruments, autonomous underwater vehicles and a tomography network. Using wireless and acoustic local area network technology one of the main objectives of the experiment was to demonstrate the combined, cordinated and adaptive use of all these systems within the new Autonomous Oceanographic Sampling Network [1] paradigm for mapping of highly dynamic ocean processes.

\section{A. Acoustic Network}

The acoustic components of the network consisted of 4 moored nodes deployed South of Stuart Island as shown in Fig. 2. Each network node consisted of a U-shaped mooring, as shown in Fig. 3, equipped with the following: GPS receiver, Radio Ethernet link to shore control station, 16 element vertical hydrophone array, $15 \mathrm{kHz}$ Acoustic Com- 


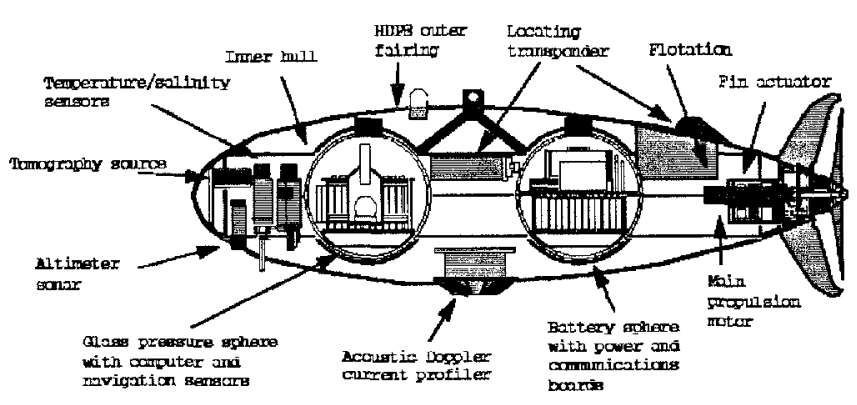

Fig. 4. Odyssey class AUV used in Haro Strait Experiment.

munications Source, $1.5 \mathrm{kHz}$ Tomography Source, Array Tracking Sources, Array Anchor with Pitch, Roll, Heading instrumentation, and Data Logging Computer with 50 Mflop DSP.

\section{B. Autonomous Underwater Vehicles}

Two Odyssey Ilb type AUVs (Fig. 4) were used for the Haro Strait experiment [2]. Derivatives of the original Odyssey design [3], the AUVs employ a fairing to obtain a low-drag configuration and are propelled by a single propulsor at the stern. The main pressure housings are glass spheres. The two vehicle used for this experiment each carried approximately $1 \mathrm{~kW}-\mathrm{hr}$ of $\mathrm{Ag}-\mathrm{Zn}$ batteries, which provided a maximum endurance of three hours at a speed of $1.6 \mathrm{~m} / \mathrm{s}$.

Measuring $2.2 \mathrm{~m}$ in length, and $0.6 \mathrm{~m}$ in diameter, the Odyssey fairing encloses a substantial wet volume for integrating various oceanographic payloads. One vehicle in this experiment carried a $300 \mathrm{kHz}$ RDI Acoustic Doppler Current Profiler, an acoustic tomography source, and a WHOI acoustic modem. The ADCP was mounted in a downward looking configuration. The second vehicle carried a $100 \mathrm{kHz}$ side-scan and $120 \mathrm{kHz}$ upward looking sonar, developed by IOS as an integrated system. Both vehicles were equipped with Sea Bird conductivity and temperature sensors.

Navigation for the AUVs was provided by a longbaseline array. The vehicles were also tracked with a commercial ultrashort base-line navigation system mounted on a DGPS equipped boat. For some of the experiments, the LBL system was used as a simple command link for sending heading commands to the vehicle from a deck unit on the tracking vessel.

\section{Floats and Drifters}

Fronts typically exhibit strong horizontal shear. We used various techniques for mapping the front and measuring the shear. Two small floats equipped with GPS navigation identified as SPORT1 and SPORT2 were deployed from a small boat (whaler) at various points in the

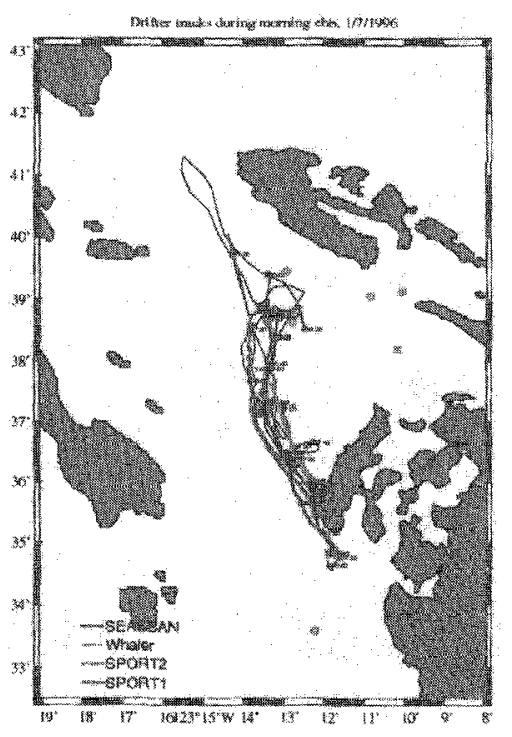

Fig. 5. Location of all GPS-tracked drifters south of Stuart Island on July 1, 1996. Deployment occurred at about 1600Z (peak ebb) and lasted until slack water. SPORT drifters were redeployed at $1800 \mathrm{Z}$. Whaler track indicates observed frontal location. Moorings are indicated as in Fig. 2

vicinity. The GPS tracks for one set of deployments is shown in Fig. 5. The whaler was also equipped with GPS positioning and was used to map the location of the front and related features by running it up and down the most obvious surface expression. Notes were made at the time on the appearance of the surface, so that the combination of GPS track and local description and be used in putting together a description of the front. In addition, the freely drifting Doppler sonar SEASCAN, which was deployed from the CSS VECTOR, was tracked by GPS (all GPS tracking was telemetered back to the ship for real-time display). SEASCAN drift tracks represent motion primarily at a depth of approximately $25 \mathrm{~m}$, since the primary drag of the instrument was at that depth, whereas the SPORT drifters moved essentially with the surface current. While detailed analysis of the drift speeds at different times will be required, it can be said at this time that the Lagrangian drifter tracks clearly indicate the differential motion of the surface water on either side of the front.

\section{RESULTS}

\section{A. Acoustic Communication and Tomography}

Several different acoustic tomography experiments were carried out, using both the $1.5 \mathrm{kHz}$ and $15 \mathrm{kHz}$ sources on the vertical arrays and and one of the AUV. Fig. 6 shows an example of the acoustic data received on one of the vertical arrays, from a transmission from the acoustic modem source at the top of the same array. The transmitted signal consistst of a 28 symbol Barker code transmitted with 


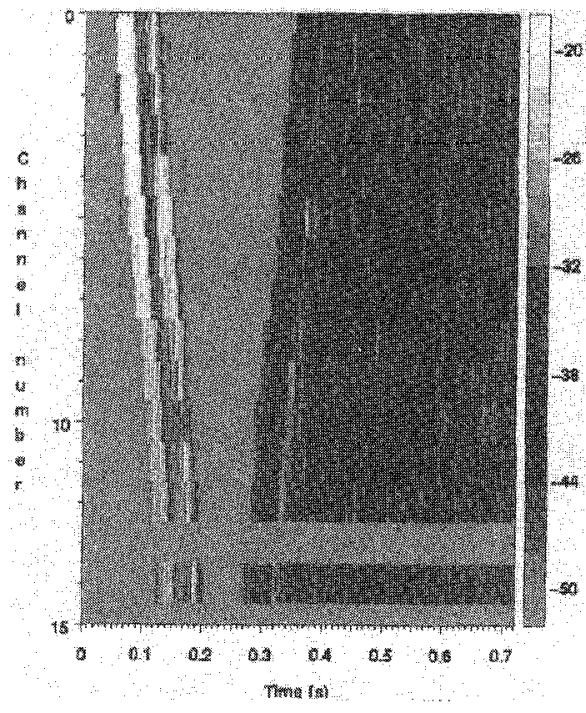

Fig. 6. Envelope of demodulated, matched filtered signals received on vertical array. 28 symbol Barker code transmitted by acoustic modem at top of array, with carrier frequency $15 \mathrm{kHz}$.

a $15 \mathrm{kHz}$ carrier frequency. The received signals have been matched filtered, and Fig. 6 shows contours of the envelope of the demodulated and match-filtered signals. The received signals show distinct direct and surface reflected paths, followed by the bottom-interacting multibles. Here it is interesting to note the relative insignificance of surface relative to the bottom reverberation in this monostatic scenario. Similar effects are seen in the bi-static tomography data.

\section{B. Side-Scan Imagery}

Following an earlier demonstration of the potential for scanning imaging sonars in such environments (Farmer, D'Asaro et al, 1995) we deployed a similar instrument during the present study. SEASCAN has four mechanically driven fan beam sonars each of which rotates $90 \mathrm{deg}$. in $30 \mathrm{sec}$ producing successive images of the sea surface from its drifting depth of $25 \mathrm{~m}$, somewhat analogous to a radar map. An example is shown in Fig. 7 in which red represents strong back scatter and blue is low back scatter. At the time this image was generated SEASCAN was in the middle of an active region of the front. Red portions of the image most likely correspond to areas of the surface roughened by small waves, many of which break producing bubble clouds, even in calm conditions. In the lower part of Fig. 7 a time series of back scatter from a vertically oriented sonar is also shown. Red patterns near the surface show bubble clouds being drawn downwards; the arrow corresponds to the time that the circular image above was made. The irregular solid blue at the bottom of the vertical sonar image corresponds to the changing depth of the instrument as it moves on its rubber cord in the presence of quite strong vertical currents.

Similar sidescan imagery was acquired from the AUV

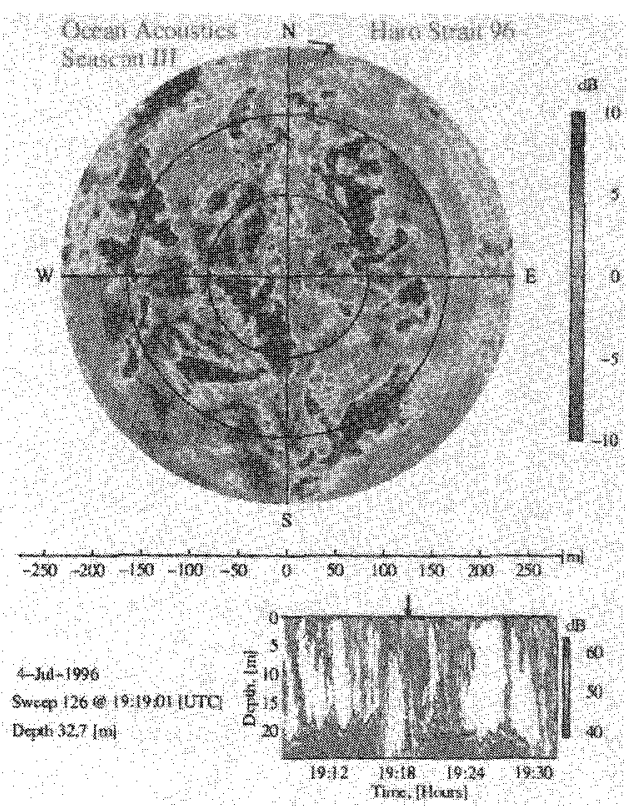

Fig. 7. Imaging sonar views of frontal structure.

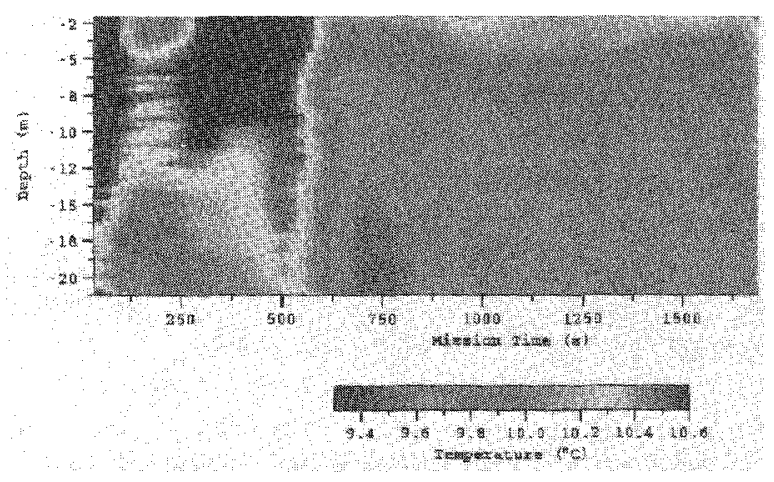

Fig. 8. CTD measurement showing frontal crossing by AUV

and one of the goals of the combined AUV/SEASCAN deployment was to acquire simultaneous surface image data from the two platforms in the same vicinity. The imaging sonars on both platforms used coherent detection allowing Doppler processing of the results.

In addition to the drifting sonar measurements, extensive ADCP and echosounder data along with CTD profiles were acquired from the CSS VECTOR both in the immediate vicinity of the AUV and tomography operational area and throughout Haro Strait.

\section{AUV Surveys}

The AUV operations in Haro Strait had four basic objectives:

- Demonstrate moving source tomography from the AUV

- Take coordinated AUV-Sea Scan Drifter data sets 


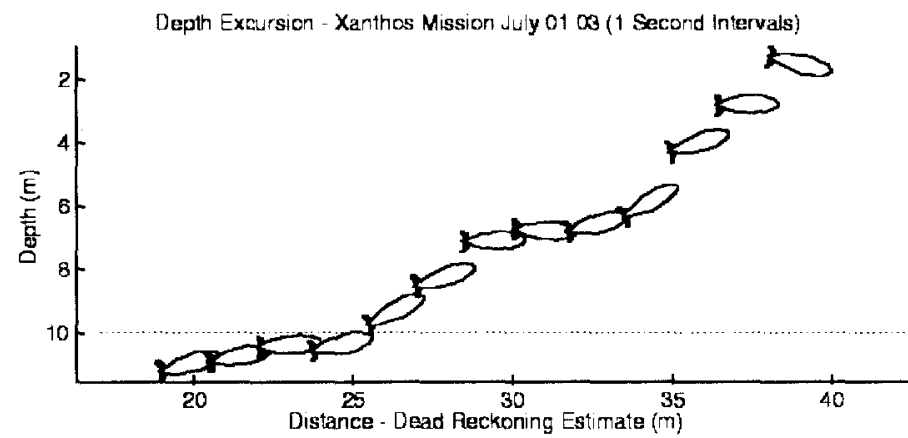

Fig. 9. AUV pitch during survey mission, with pitch indicating attempt of compensating for large vertical velocities.

- Demonstrate model driven sampling with Harvard group data

- Take coordinated ADCP/CTD data sets for fast synoptic mapping test.

All four of the these objectives were met. Fig. 8 shows data for one of the 'front imaging experiments', in which AUV runs were designed to test model predictions of front location. For this experiment, the vehicle flew a yo-yo trajectory between 2 and 20 meters, completing 34 complete yo-yo cycles. Heading of the vehicle during the run was $90 \mathrm{M}$ for the first 1200 seconds of the mission, followed by 900 seconds on a reciprocal heading. The trajectory carried the vehicle across the front twice, as illustrated by the strong horizontal temperature contrast observed at approximated 600 and 1700 seconds.

The more powerful fronts observed in the experiment had a strong influence on vehicle trajectory. Fig. 9 shows the vehicle path during a mission in which the vehicle was attempting to hold a constant $10 \mathrm{~m}$ depth. Usually the vehicle is able to hold depth to within $0.2 \mathrm{~m}$. However, this data set shows the vehicle apparently flying into a strong upwelling event. The event both deflect the vehicle to a pitch-up orientation, and physically lifts the vehicle to the surface. The maximum vertical velocity observed here is approximately $1.5 \mathrm{~m} / \mathrm{s}$.

Overall, AUV performance in Haro Strait was very reliable. Over 86 vehicle missions were run with no vehicle failures.

\section{CONCLUSION}

The June 1996 Haro Strait experiment demonstrated the feasibility of using a network of small AUVs in conjunction with acoustic tomography and other oceanographic techniques to measure the volumetric properties of estuarian and tidal driven coastal front systems. Even though the closed-loop connectivity of all the various measurement systems of the hybryd oceanographic sampling network is yet to be achieved, the experiment demonstrated the feasibility of the concept by performing several combined experiments. Thus, the use of the AUVs for moving source tomography was demonstrated, and also the adaptive operation of multible AUVs in coordination with a free drifting oceanographic instrument was demonstrated. On the other hand, the experiment identified several fundamental, scientific and technological issues yet to be resolved before the new measurement concept can become of routine use. For example, the acoustic environment in such regions is a significant complicating factor, with extremely high ambient noise levels due to shipping and boating, and the extremely dynamic oceanography yields high scintillation indices. The operation of acoustic systems for communication and navigation is therefore non-trivial, and the experiment clearly identified a need for more robust acoustic systems and processing algorithms.

\section{ACKNOWLEDGMENT}

This work was supported by the Office of Naval Research, Code 321HL, under the Frontal Dynamics PRIMER.

\section{REFERENCES}

[1] T.B. Curtin, J.G. Bellingham, J.Catipovic and D. Webb, Autonomous Oceanographic Sampling Networks, Oceanography, Vol. 6, No. 3, 86-94. 1993.

2] T.W.Altshuler, T.W.Vanek, and J.G. Bellingham, Odyssey 11 b - Towards Commercialization of AUVs, Sea Technology, December 1995, 15-19.

[3] J.G. Bellingham, C.A. Goudey, T.R. Consi, and C. Chryssostomidis, A Small, Long-Range Vehicle for Deep Ocean Exploration, Proceedings of the Second International Offshore and Polar Engineering Conference, San Francisco, Vol. 2, 461-467, 1992.

[4] M.G.G. Foreman, R. A. Walters, R. F. Henry, C. P. Kellar, A. G. Dolling, A tidal model for eastern Juan de Fuca Strait and the southern Strait of Georgia, J. Geophys. Res. 100 (C1), $721-740,1995$.

[5] D. M. Farmer, E. D'Asaro, M. V. Trevorrow, G. T. Dairiki, Three dimensional structure in a tidal convergence front, Cont. Shelf Res. 15 (13), 1649-1673, 1995. 